01,11

\title{
Эффективные коэффициенты диффузии и термическая устойчивость структуры металлического стекла $\mathrm{Fe}_{48} \mathrm{Co}_{32} \mathrm{P}_{14} \mathrm{~B}_{6}$
}

\author{
(C) С.В. Васильев ${ }^{1,2}$, В.И. Парфений ${ }^{1,2}$, Е.А. Першина ${ }^{3}$, А.С. Аронин ${ }^{3}$, О.В. Коваленко ${ }^{1}$, В.И. Ткач ${ }^{1}$ \\ ${ }^{1}$ Донецкий фозико-технический институт им. А.А. Галкина, \\ Донецк, Украина \\ ${ }^{2}$ Донбасская национальная академия строительства и архитектуры, \\ Макеевка, Украина \\ ${ }^{3}$ Институт фризики твердого тела РАН, \\ Черноголовка, Московская обл., Россия \\ E-mail: stalker_1345@mail.ru
}

Поступила в Редакцию 30 июня 2020 г.

В окончательной редакции 18 июля 2020 г.

Принята к публикации 27 июля 2020 г.

По результатам электронномикроскопических исследований частично закристаллизованных образцов металлического стекла $\mathrm{Fe}_{48} \mathrm{Co}_{32} \mathrm{P}_{14} \mathrm{~B}_{6}$ впервые определены значения скорости линейного роста эвтектических колоний в диапазоне температур $679-717 \mathrm{~K}$. По этим данным в рамках классической теории кристаллизации рассчитаны значения эффективных коэффициентов диффузии, контролирующей рост. Установлено, что температурная зависимость эффективных коэффициентов диффузии в стекле $\mathrm{Fe}_{48} \mathrm{Co}_{32} \mathrm{P}_{14} \mathrm{~B}_{6}$ хорошо аппроксимируется уравнением типа Аррениуса, а их значения в диапазоне температур кристаллизации стекла оказались примерно на 2-3 порядка величины ниже, чем в промышленном стекле $\mathrm{Fe}_{40} \mathrm{Ni}_{40} \mathrm{P}_{14} \mathrm{~B}_{6}$ Определены значения эффективного коэффициента диффузии и объемной плотности кристаллитов при температуре начала кристаллизации, найдена корреляция между предэкспоненциальными множителями и энергиями активации. Показано, что параметры, характеризующие эффективную диффузию в исследованном стекле, хорошо согласуются с опубликованными данными для эвтектически кристаллизующихся металлических стекол. Установлено, что физической причиной повышенной термической устойчивости структуры железокобальтового стекла по сравнению с $\mathrm{Fe}_{40} \mathrm{Ni}_{40} \mathrm{P}_{14} \mathrm{~B}_{6}$ является более низкая подвижность атомов на межфазной границе стекло/кристалл.

Ключевые слова: металлическое стекло, термическая устойчивость, линейный рост, эффективный коэффициент диффузии, объемная плотность кристаллов.

DOI: $10.21883 /$ FTT.2020.12.50203.140

\section{1. Введение}

В многочисленных исследованиях процесса кристаллизации металлических стекол (МС) важную роль играет фундаментальный аспект, основными задачами которого является установление факторов (величин), контролирующих термическую устойчивость аморфного состояния, и численная оценка термодинамических и кинетических параметров, определяющих скорости зарождения и роста кристаллов. В свою очередь, сравнительный анализ значений параметров процесса кристаллизации МС различного химического состава открывает возможности для выяснения физической природы эмпирически установленных эффектов легирования на термическую устойчивость аморфного состояния и микроструктуру закристаллизованных образцов.

Наиболее важным кинетическим параметром, во многом определяющим термическую устойчивость МС и параметры структуры стекол на начальных стадиях кристаллизации, является эффективный коэффициент диффузии, $D_{\text {eff }}[1]$. В отличие от коэффициентов само- и гетеродиффузии, которые характеризуют процесс атомных блужданий в кристаллической решетке или аморфной матрице, параметр $D_{\text {eff }}$ характеризует скорость перехода атомов через межфазную границу в процессах зарождения и роста кристаллов. Физическая природа этого параметра и методы оценки подвижности атомов на межфазной границе являются предметом анализа еще с середины прошлого столетия [2]. Однако результаты проведенных до настоящего времени теоретических исследований и моделирования $[3,4]$ показывают, что проблема априорной оценки значений $D_{\text {eff }}$ остается нерешенной даже для чистых металлов.

С другой стороны, поскольку коэффициент диффузии входит в уравнения, описывающие скорости зарождения и роста кристаллов, то представлялось интересным оценить его значения из сопоставления экспериментально измеренных и рассчитанных параметров кристаллизации. Такой подход был использован в работе [5], в которой значения $D_{\text {eff }}$ для ряда МС на основе системы $\mathrm{Fe}-\mathrm{B}$ были определены по изменениям размеров, $r$, кристаллов $\alpha$-Fe, растущих по параболическому закону Зинера [6] $r=\alpha \sqrt{D_{\text {eff }} t}$ (где $\alpha-$ безразмерная величина порядка единицы, зависящая от состава растущих кристаллов и матрицы, а $t$ - время). Анализ полученных результатов показал, что значения $D_{\text {eff }}$, оцененные по ро- 
сту, оказались близкими к коэффициентам диффузии бора в кристаллах $\alpha$-Fe и зернограничной диффузии атомов железа [6]. В принципе это неудивительно, поскольку рост кристаллов $\alpha$-Fe происходит путем перехода через межфазную границу преимущественно атомов железа и оттеснения атомов бора в аморфную матрицу. Однако аналогичный анализ, проведенный по росту кристаллов $(\mathrm{Fe}, \mathrm{Ni})_{3}(\mathrm{P}, \mathrm{B})$ в $\mathrm{MC} \mathrm{Fe}_{40} \mathrm{Ni}_{40} \mathrm{P}_{14} \mathrm{~B}_{6}$, показал [7], что значения $D_{\text {eff }}$ существенно отличаются от коэффициентов гетеродиффузии легирующих элементов в этом стекле.

Эти и другие [8] примеры относятся к анализу процесса первичного (или диффузионно-контролируемого) роста [6], когда состав растущих кристаллов отличается от состава материнской (аморфной) фазы. Однако во многих МС рост кристаллов происходит по эвтектическому или полиморфному механизмам (линейный рост), скорость которого контролируется диффузией на межфазной границе [9]. Очевидно, что природа эффективных коэффициентов диффузии, входящих в уравнения эвтектического роста кристаллов, отличается от природы коэффициентов, контролирующих рост первичных кристаллов, хотя принципы оценки их значений по скорости роста [10], структуре закристаллизованных образцов $[11,12]$ или изменениям доли закристаллизованного объема [13] аналогичны.

Поскольку эффективные коэффициенты диффузии определяют скорости зарождения и роста кристаллов, их значения должны быть тесно связаны с термической устойчивостью структуры МС. Действительно, недавно проведенный анализ [1] показал, что значения Deff при температурах начала кристаллизации $\mathrm{MC}, T_{\text {ons }}$, (пороговые значения) лежат в относительно узком диапазоне значений от $3 \cdot 10^{-18}$ до $1.7 \cdot 10^{-20} \mathrm{~m}^{2} / \mathrm{s}$. Более того, расчеты показали, что плотность кристаллов, образовавшихся в $\mathrm{MC}$ в процессе нагрева до $T_{\text {оns }}$, является убывающей функцией пороговых значений $D_{\text {eff. }}$ Однако перечисленные выводы были сделаны по результатам анализа начальных стадий первичной кристаллизации, поскольку температурные зависимости эффективных коэффициентов диффузии, контролирующей эвтектический и полиморфный рост, определены для ограниченного круга металлических стекол [1].

Целью настоящей работы являлось установление температурной зависимости $D_{\text {eff, }}$ контролирующей процесс роста эвтектических кристаллов в $\mathrm{MC} \mathrm{Fe}_{48} \mathrm{Co}_{32} \mathrm{P}_{14} \mathrm{~B}_{6}$. Выбранный в качестве объекта исследования сплав обладает наибольшей индукцией насыщения $(1.42 \mathrm{~T})$ в группе магнитно-мягких стекол $\mathrm{Fe}_{80-x} \mathrm{Co}_{x} \mathrm{P}_{14} \mathrm{~B}_{6}(20 \leq x \leq 40)$, синтезированных в 2004 г. [14]. Помимо высокой индукции насыщения это стекло характеризуется более высокими значениями температур Кюри и кристаллизации [15] по сравнению с промышленным МС $\mathrm{Fe}_{40} \mathrm{Ni}_{40} \mathrm{P}_{14} \mathrm{~B}_{6}$. Высокий уровень важных с практической точки зрения физических свойств является стимулом для количественного анализа параметров, определяющих температурно-временные границы устойчивости структуры стекла $\mathrm{Fe}_{48} \mathrm{Co}_{32} \mathrm{P}_{14} \mathrm{~B}_{6}$. Проведенный недавно анализ показал [16], что более высокая термическая устойчивость $\mathrm{MC} \mathrm{Fe}_{48} \mathrm{Co}_{32} \mathrm{P}_{14} \mathrm{~B}_{6}$ по сравнению со стеклом $\mathrm{Fe}_{40} \mathrm{Ni}_{40} \mathrm{P}_{14} \mathrm{~B}_{6}$ обусловлена более низким характеристическим временем кристаллизации и более сильным отклонением скорости зарождения от стационарного значения. Однако оба параметра (характеристические времена кристаллизации и нестационарности), количественно оцененные в [16], являются феноменологическими и не описывают конкретные причины различия термической устойчивости $\mathrm{MC}$ на $\mathrm{FeNi}-$ и $\mathrm{FeCo-основе.}$

В отличие от [16] в настоящей работе проведены количественные оценки одного из важнейших кинетических параметров процесса кристаллизации МС $\mathrm{Fe}_{48} \mathrm{Co}_{32} \mathrm{P}_{14} \mathrm{~B}_{6}$ - эффективного коэффициента диффузии. Основой для оценок $D_{\text {eff }}$ послужили впервые экспериментально определенные значения скорости роста кристаллов (эвтектических колоний) при различных температурах. Полученные в работе параметры сравнивались с параметрами, характеризующими процесс кристаллизации $\mathrm{MC} \mathrm{Fe}_{40} \mathrm{Ni}_{40} \mathrm{P}_{14} \mathrm{~B}_{6}$.

\section{2. Методы получения и исследования структуры образцов}

Металлическое стекло номинального состава $\mathrm{Fe}_{48} \mathrm{Co}_{32} \mathrm{P}_{14} \mathrm{~B}_{6}$ было получено в форме ленты толщиной $27 \mu \mathrm{m}$ и шириной $1.5 \mathrm{~mm}$ методом спиннингования расплава на внешнюю поверхность вращающегося медного валка. Более подробно методика приготовления этого сплава и параметры процесса литья описаны в работах $[14,16]$. Термографические исследования полученного индукционным плавлением слитка, показали, что он плавится в одну стадию при температуре $1265 \pm 3 \mathrm{~K}$, что в пределах погрешности совпадает с температурой плавления сплава $\mathrm{Fe}_{40} \mathrm{Co}_{40} \mathrm{P}_{14} \mathrm{~B}_{6}(1262 \pm 3 \mathrm{~K}) \quad$ [12]. Аморфный характер структуры быстроохлажденных лент и двухфазное строение $\left(\alpha-\mathrm{FeCo}+(\mathrm{FeCo})_{3}(\mathrm{~PB})\right)$ закристаллизованных образцов были установлены рентгенографически [16]. Параметры термической устойчивости структуры в условиях нагрева со скоростью $0.167 \mathrm{~K} / \mathrm{s}$ были установлены по термограмме, полученной методом дифференциальной сканирующей калориметрии (ДСК) на калориметре Perkin-Elmer DSC-7.

Скорость роста кристаллов (колоний) оценивалась по результатам электронномикроскопических исследований „на просвет“ (JЕM-100CX при ускоряющем напряжении $100 \mathrm{kV}$ ) структуры образцов, частично закристаллизованных в изотермических условиях при различных температурах. Режимы термообработки выбирались по результатам анализа изотермической кристаллизации аморфных лент в диапазоне температур 687-714 K [16] таким образом, чтобы доля закристаллизованного объема не превышала $20 \%$, т.е. обеспечивались условия преимущественно свободного роста кристаллов без столкновений. Для снижения времени прогрева отжиги ленточных образцов при температурах 679, 696 и $717 \mathrm{~K}$ $( \pm 1 \mathrm{~K})$ в течение 5960,1156 и $107 \mathrm{~s}$ соответственно были проведены в ванне из расплавленных солей. 
Фольги для просвечивающей электронной микроскопии готовились ионным травлением. Скорости роста при различных температурах оценивались по площади кристаллита наибольшего размера, обнаруженного на серии микрофотографий, в предположении, что кристаллит рос с момента начала отжига, а наблюдаемое сечение проходит через его центр [10].

\section{3. Результаты и обсуждение}

Электронномикроскопические исследования структуры термообработанных образцов показали, что даже в отсутствие столкновений форма растущих кристаллов с размерами, превышающими 40-50 nm, начинает отклоняться от сферической, что, в частности, наблюдалось и для колоний, растущих в $\mathrm{MC} \mathrm{Fe}_{80} \mathrm{~B}_{20}$ [10] и $\mathrm{Fe}_{40} \mathrm{Ni}_{40} \mathrm{P}_{14} \mathrm{~B}_{6}$ [11]. Для наиболее крупных кристаллитов с размерами выше $200 \mathrm{~nm}$, которые наблюдались в исследуемых термообработанных образцах, отклонения формы от сферичности становятся существенными (рис. 1). Ввиду этого, эффективные радиусы таких кристаллитов определялись из соотношения $r=(S / \pi)^{1 / 2}$, где $S$ - площадь кристаллита. Обработка изображения, приведенного на рис. 1, дала значение $r=384 \mathrm{~nm}$. Отметим, что оценки размера по площади практически совпадают со средним значением серии радиусов, проведенных из центра описанной вокруг кристаллита окружности. Как видно из рис. 1, кристаллит имеет сложную внутреннее строение, что характерно для эвтектических колоний, формирующихся в металлических стеклах [10-12], и согласуется с установленным рентгенографически двухфазным характером структуры закристаллизованных образцов этого стекла [14]. Аналогичный характер микроструктуры имели образцы, отожженные при более низких температурах, а оценки радиусов наиболее крупных эвтектических колоний, сформированных при температурах 679 и $696 \mathrm{~K}$, составили 231 и $298 \mathrm{~nm}$ соответственно.

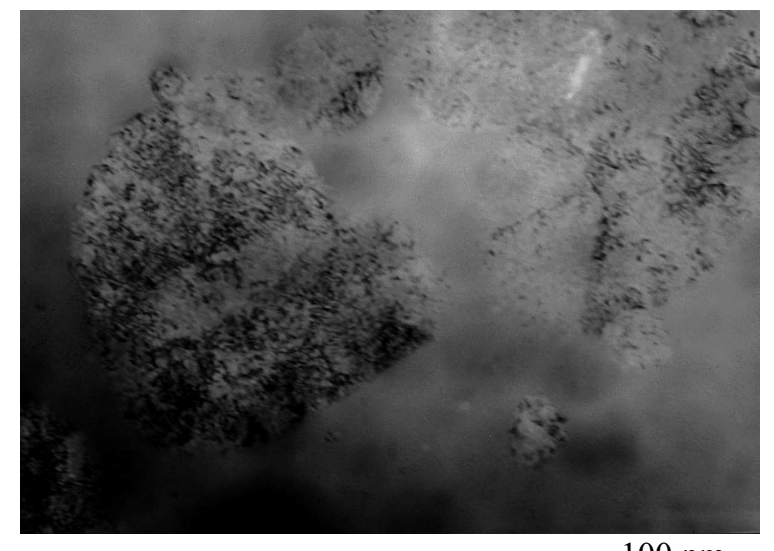

$100 \mathrm{~nm}$

Рис. 1. Эвтектическая колония наибольшего размера, сформированная в ленте $\mathrm{MC} \mathrm{Fe}_{48} \mathrm{Co}_{32} \mathrm{P}_{14} \mathrm{~B}_{6}$ при температуре $717 \mathrm{~K}$ в течение $107 \mathrm{~s}$.
Как известно [9], рост эвтектических колоний контролируется диффузией на границе раздела фаз и его скорость, $U$, в изотермических условиях постоянна, т.е. размер колонии является линейной функцией времени $(r=U t)$. Исходя из этого и приведенных выше данных, оценки скорости роста эвтектических колоний в металлическом стекле $\mathrm{Fe}_{48} \mathrm{Co}_{32} \mathrm{P}_{14} \mathrm{~B}_{6}$ при температурах 679,696 и $717 \mathrm{~K}$ дали значения $3.9 \cdot 10^{-11}, 2.6 \cdot 10^{-10}$ и $3.6 \cdot 10^{-9} \mathrm{~m} / \mathrm{s}$ соответственно. Безусловно, скорость роста кристаллов в каждом МС зависит от индивидуальных значений термодинамических и кинетических параметров, тем не менее, отметим, что скорость роста эвтектических колоний в стекле $\mathrm{Fe}_{80} \mathrm{~B}_{20}$ при температуре $673 \mathrm{~K}$, определенная по аналогичной методике $\left(3.3 \cdot 10^{-10} \mathrm{~m} / \mathrm{s}\right)$, лежит в том диапазоне значений.

Согласно классической теоретической модели [3,9], описывающей рост кристалла (эвтектической колонии), контролируемый диффузией на фронте, основной вклад в температурную зависимость вносит эффективный коэффициент диффузии

$$
U(T)=\left[D(t) / a_{0}\right] F(T),
$$

где $a_{0}$ - длина диффузионного скачка, принимаемая равной среднему атомному диаметру, а $F(T)$ - термодинамический сомножитель, равный $\{1-\exp [-\Delta G(T / R T)]\}$, в котором $\Delta G-$ молярная разность термодинамических потенциалов между материнской и кристаллической фазами, а $R$ - универсальная газовая постоянная. Для оценки значений эффективного коэффициента диффузии из соотношения (1) необходимые для этого разности термодинамических потенциалов в условиях, далеких от равновесия, рассчитывались в приближении Томсона-Спейпена [17]:

$$
\Delta G(T)=\frac{2 \Delta H_{m} T\left(T_{m}-T\right)}{T_{m}\left(T_{m}+T\right)},
$$

где $\Delta H_{m}$ и $T_{m}-$ молярная теплота и температура плавления соответственно. Корректность простого соотношения (2) была подтверждена в ряде работ (напр., [18]) и оно использовалось в работах [12,13], что позволяет сравнивать результаты, полученные в настоящей работе, с ранее опубликованными данными. Ввиду того, что теплота плавления для сплава $\mathrm{Fe}_{48} \mathrm{Co}_{32} \mathrm{P}_{14} \mathrm{~B}_{6}$ неизвестна, ее значение в настоящей работе было оценено, как $\Delta H_{m}=R T_{m}$, аналогично соотношению этих параметров для аналогично кристаллизующегося сплава Metglas 2826 [11]. Подстановка в соотношение (1) значений $T_{m}=1265 \mathrm{~K}$, $a_{0}=2.38 \cdot 10^{-10} \mathrm{~m}[13]$ и скоростей роста показала, что в диапазоне температур 679-717 K эффективные коэффициенты диффузии изменяются в пределах двух порядков величины (от $2.04 \cdot 10^{-20}$ до $2.02 \cdot 10^{-18} \mathrm{~m}^{2} / \mathrm{s}$ ) и хорошо аппроксимируются уравнением типа Аррениуса (рис. 2) $\ln D_{\text {eff }}=\exp (41.46 \pm 4)-(58984 \pm 2836) / T$. Примечательно, что энергия активации эффективной диффузии, контролирующей рост, заметно выше 

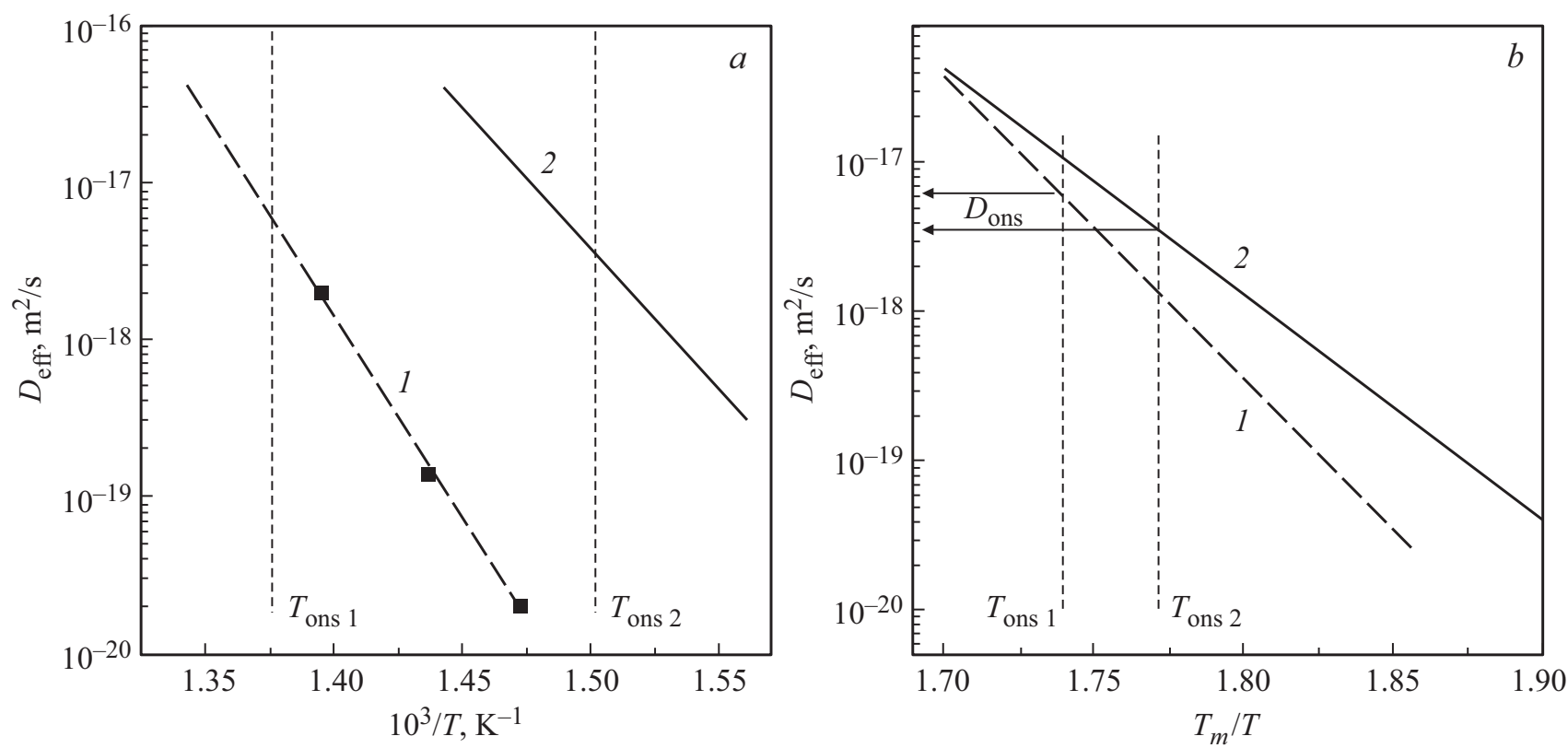

Рис. 2. Эффективные коэффициенты диффузии, контролирующей рост эвтектических колоний в металлических стеклах $\mathrm{Fe}_{48} \mathrm{Co}_{32} \mathrm{P}_{14} \mathrm{~B}_{6}(1)$ и $\mathrm{Fe}_{40} \mathrm{Ni}_{40} \mathrm{P}_{14} \mathrm{~B}_{6}(2)[13]$ в зависимости от обратной (a) и приведенной обратной температуры $(b)$. Вертикальными линиями показаны температуры начала кристаллизации стекол при нагреве со скоростью $0.167 \mathrm{~K} / \mathrm{s}$.

энергии активации кристаллизации $\mathrm{MC} \mathrm{Fe}_{48} \mathrm{Co}_{32} \mathrm{P}_{14} \mathrm{~B}_{6}$ $(48000 \mathrm{~K}[16])$, определенной по широко используемому в литературе методу Киссинджера [19], и энергии активации характерного времени кристаллизации $(44800 \mathrm{~K})[16]$. Различия энергий активации, характеризующих процессы кристаллизации МС и эффективной диффузии, наблюдались в ряде работ $[12,13,20]$, и, по всей видимости, обусловлены нестационарным характером процесса зарождения.

В свою очередь, установленная температурная зависимость $D_{\text {eff }}(T)$ позволяет оценить пороговое значение эффективного коэффициента диффузии при температуре начала кристаллизации. Для этой цели в работе была проанализирована термограмма ДСК стекла $\mathrm{Fe}_{48} \mathrm{Co}_{32} \mathrm{P}_{14} \mathrm{~B}_{6}$, измеренная при скорости нагрева $0.167 \mathrm{~K} / \mathrm{s}$, поскольку большинство опубликованных до настоящего времени оценок было получено при таких условиях [1]. Стандартный анализ профиля скорости кристаллизации на термограмме ДСК показал, что температура начала кристаллизации (точка пересечения касательной в точке перегиба с фоном) стекла $\mathrm{Fe}_{48} \mathrm{Co}_{32} \mathrm{P}_{14} \mathrm{~B}_{6}$ составляет $727 \mathrm{~K}$ (рис. 3). Из сопоставления этой температуры с кинетической кривой кристаллизации $X(T)$, полученной численным интегрированием сигнала ДСК (сплошная кривая на рис. 2), следует, что доля превращенного объема в исследуемом стекле при $T_{\text {ons }}\left(X_{\text {ons }}\right)$ составляет 0.055 (с погрешностью не более $15 \%$ ). Использованный метод оценки $X_{\text {ons }}$ по кинетическим данным представляется полезным, поскольку анализ показал [1], что определенные таким образом доли закристаллизованного объема для различных механизмов кристаллизации не проявляются визуально на дифракционных картинах.
Сопоставление параметров $T_{\text {ons }}$ и $X_{\text {ons }}$, характеризующих термическую устойчивость $\mathrm{MC} \mathrm{Fe}_{48} \mathrm{Co}_{32} \mathrm{P}_{14} \mathrm{~B}_{6}$, с аналогично определенными характеристиками стекла $\mathrm{Fe}_{40} \mathrm{Ni}_{40} \mathrm{P}_{14} \mathrm{~B}_{6}$ [1] показало, что температура начала кристаллизации $\mathrm{FeNi}$ существенно ниже и составляет $666 \mathrm{~K}$, в то время как доля $X_{\text {ons }}$ примерно одинакова $(0.05)$. Как отмечалось выше, феноменологический анализ различий термической устойчивости стекол

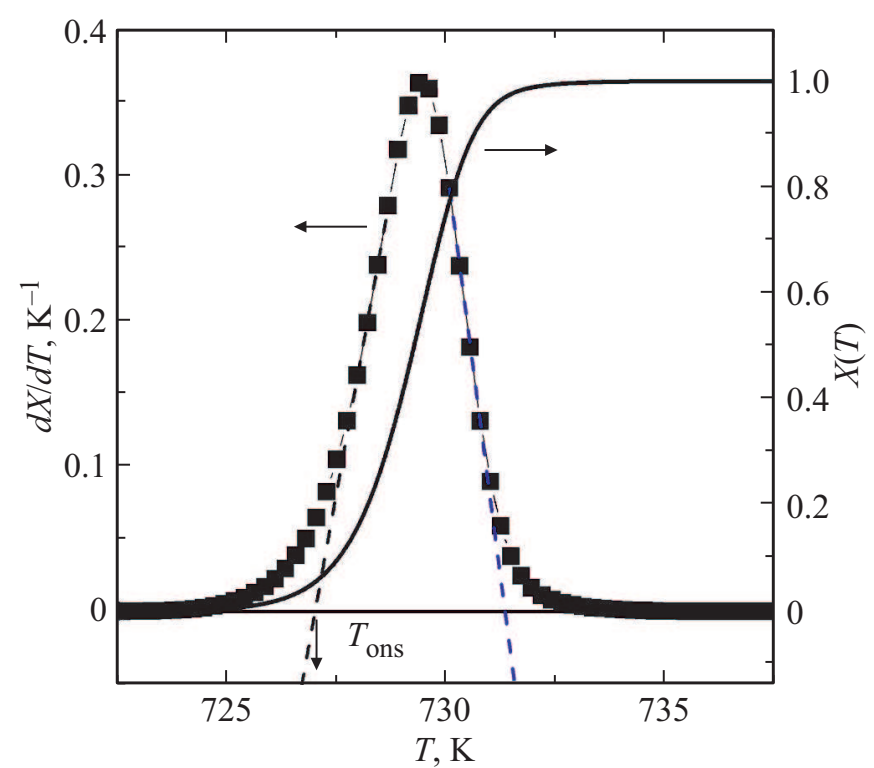

Рис. 3. Оценка температуры начала кристаллизации и доли превращенного объема при $T_{\text {ons }}\left(X_{\text {ons }}\right)$ стекла $\mathrm{Fe}_{48} \mathrm{Co}_{32} \mathrm{P}_{14} \mathrm{~B}_{6}$ по термограмме ДСК, снятой при скорости нагрева $0.167 \mathrm{~K} / \mathrm{s}$ $\left(T_{\mathrm{ons}}=727 \mathrm{~K}, X_{\mathrm{ons}}=0.055\right)$. 
Температуры начала кристаллизации, параметры аррениусовских зависимостей $D_{\text {eff }}(T)$, их пороговые значения и плотность кристаллов при температурах начала кристаллизации МC, кристаллизующихся по эвтектическому механизму

\begin{tabular}{c|c|c|c|c|c|c|c|c}
\hline № & Сплав & $T_{\text {опs }}, \mathrm{K}$ & $X_{\text {оns }}$ & $D_{0}, \mathrm{~m}^{2} / \mathrm{s}$ & $Q, \mathrm{~K}$ & $D_{\text {eff }}\left(T_{\text {оns }}\right), \mathrm{m}^{2} / \mathrm{s}$ & $N_{\text {опs, }}, \mathrm{m}^{3}$ & Источник \\
\hline 1 & $\mathrm{Fe}_{80} \mathrm{~B}_{20}$ & 698 & 0.04 & 71.8 & 31900 & $1.0 \cdot 10^{-18}$ & $9.9 \cdot 10^{17}$ & {$[21]$} \\
2 & $\mathrm{Fe}_{40} \mathrm{Co}_{40} \mathrm{P}_{14} \mathrm{~B}_{6}$ & 730 & 0.05 & $6.4 \cdot 10^{16}$ & 57800 & $2.6 \cdot 1^{-18}$ & $4.2 \cdot 10^{17}$ & {$[21]$} \\
3 & $\mathrm{Fe}_{40} \mathrm{Ni}_{40} \mathrm{P}_{14} \mathrm{~B}_{6}$ & 666 & 0.05 & $2.3 \cdot 10^{9}$ & 41120 & $3.5 \cdot 10^{-18}$ & $9.8 \cdot 10^{16}$ & {$[13]$} \\
4 & $\mathrm{Fe}_{48} \mathrm{Co}_{32} \mathrm{P}_{14} \mathrm{~B}_{6}$ & 727 & 0.055 & $1.01 \cdot 10^{18}$ & 58984 & $5.89 \cdot 10^{-18}$ & $7.9 \cdot 10^{16}$ &
\end{tabular}

$\mathrm{Fe}_{48} \mathrm{Co}_{32} \mathrm{P}_{14} \mathrm{~B}_{6}$ и $\mathrm{Fe}_{40} \mathrm{Ni}_{40} \mathrm{P}_{14} \mathrm{~B}_{6}$ показал, в частности [16], что характеристическое время кристаллизации $\mathrm{FeCo}$ стекла выше. Поскольку величина характеристического времени обратно пропорциональна скоростям зарождения и роста кристаллов, которые контролируются эффективной диффузией, то представлялось интересным сравнить непосредственно величины $D_{\text {eff }}$ для этих стекол. В результате анализа процесса кристаллизации МC $\mathrm{Fe}_{40} \mathrm{Ni}_{40} \mathrm{P}_{14} \mathrm{~B}_{6}$ в широком диапазоне скоростей нагрева было установлено [13], что температурная зависимость эффективного коэффициента диффузии удовлетворительно аппроксимируется уравнением $D_{\text {eff }}^{\mathrm{FeNi}}=2.3 \cdot 10^{9} \exp (-41120 / T), \mathrm{m}^{2} / \mathrm{s}$. Рассчитанные по этому соотношению в диапазоне температур кристаллизации стекла $\mathrm{Fe}_{40} \mathrm{Ni}_{40} \mathrm{P}_{14} \mathrm{~B}_{6}(645-675 \mathrm{~K})$ значения $D_{\text {eff }}$ показаны сплошной линией на рис. 2,a. Сопоставление линейных экстраполяций показанных на рис. 2,a зависимостей в области температур кристаллизации МС $\mathrm{Fe}_{48} \mathrm{Co}_{32} \mathrm{P}_{14} \mathrm{~B}_{6}$ и $\mathrm{Fe}_{40} \mathrm{Ni}_{40} \mathrm{P}_{14} \mathrm{~B}_{6}$ показывает, что величина $D_{\text {eff }}$ FeCo стекла примерно на 2-3 порядка ниже, чем стекла на железоникелевой основе. Примечательно, что нормировка температурной шкалы на температуры плавления этих сплавов $\left(T_{m}\right.$ сплава $\mathrm{Fe}_{40} \mathrm{Ni}_{40} \mathrm{P}_{14} \mathrm{~B}_{6}$ была взята равной $1180 \mathrm{~K}[13]$ ) не меняет характера взаимного расположения зависимостей $D_{\text {eff }}(T)$, рис. $2, b$. Таким образом, из проведенного анализа следует, что основной причиной повышенной термической устойчивости МС $\mathrm{Fe}_{48} \mathrm{Co}_{32} \mathrm{P}_{14} \mathrm{~B}_{6}$ по сравнению с $\mathrm{Fe}_{40} \mathrm{Ni}_{40} \mathrm{P}_{14} \mathrm{~B}_{6}$, является значительно более низкая подвижность атомов на границе раздела растущих кристаллов.

Найденная в настоящей работе температурная завизсимость $D_{\text {eff }}(T)$ для $\mathrm{MC} \mathrm{Fe}_{48} \mathrm{Co}_{32} \mathrm{P}_{14} \mathrm{~B}_{6}$ позволила оценить значение эффективного коэффициента при температуре начала кристаллизации $(727 \mathrm{~K})$, которое составило $5.9 \cdot 10^{-18} \mathrm{~m}^{2} / \mathrm{s}$. Аналогичная оценка порогового значения $D_{\text {eff }}\left(T_{\text {ons }}\right)$ для $\mathrm{MC} \mathrm{Fe}_{40} \mathrm{Ni}_{40} \mathrm{P}_{14} \mathrm{~B}_{6}$ (при $666 \mathrm{~K})$ по приведенной выше зависимости $D_{\text {eff }}(T)$ из работы [13] дала близкое значение $\left(3.5 \cdot 10^{-18} \mathrm{~m}^{2} / \mathrm{s}\right)$, что схематично показано на рис. 2. Отметим, что, несмотря на отличие полученной в работе [13] зависимости $D_{\text {eff }}(T)$ для стекла $\mathrm{Fe}_{40} \mathrm{Ni}_{40} \mathrm{P}_{14} \mathrm{~B}_{6}$ от опубликованной ранее $[21] \quad D_{\mathrm{eff}}^{\mathrm{FeNi}}=1.1 \cdot 10^{11} \exp (-43800 / T)$, значения эффективных коэффициентов в диапазоне температур кристаллизации этого стекла практически не отличаются от приведенных на рис. 2, как и пороговое значение $3.0 \cdot 10^{-18} \mathrm{~m}^{2} / \mathrm{s}$, рассчитанное из этой зависимости $[1]$.

Температурная зависимость $D_{\text {eff }}(T)$ и экспериментально оцененная величина доли кристалличности при температуре начала кристаллизации позволяют оценить средние размеры $\left(L_{\text {ons }}\right)$ и плотность $\left(N_{\text {ons }}\right)$ кристаллитов, сформированных в $\mathrm{MC} \mathrm{Fe}_{48} \mathrm{Co}_{32} \mathrm{P}_{14} \mathrm{~B}_{6}$ в процессе нагрева до $T_{\text {ons }}[1]$. Плотность кристаллтов оценивалась из соотношения $N_{\text {ons }}=6 X_{\text {ons }} /\left(\pi L_{\text {ons }}^{3}\right)$, а размер колонии, линейно растущей в условиях нагрева, определялся как $L_{\text {ons }}=2 U\left(T_{\text {ons }}\right) t_{\text {eff }}\left(T_{\text {ons }}\right)$, где $t_{\text {eff }}\left(T_{\text {ons }}\right)=T_{\text {ons }}^{2} /(q Q)-$ эффективное время диффузионно-контролируемого процесса с энергией активации $Q$ при нагреве со скоростью $q$ [21]. Расчеты показали, что экспериментально оцененная доля кристалличности при $T_{\mathrm{ons}}$, равная 0.055 , создается в $\mathrm{MC} \mathrm{Fe}_{48} \mathrm{Co}_{32} \mathrm{P}_{14} \mathrm{~B}_{6}$ в процессе нагрева кристаллитами, средний размер которых достигает $1.1 \mu \mathrm{m}$, а объемная плотность $-7.9 \cdot 10^{16} \mathrm{~m}^{3}$.

Представлялось интересным сравнить полученное значение объемной плотности кристаллитов в МС $\mathrm{Fe}_{48} \mathrm{Co}_{32} \mathrm{P}_{14} \mathrm{~B}_{6}$ с аналогичными структурными характеристиками других стекол, кристаллизующихся по эвтектическому механизму. Такая попытка была сделана в работе [1] для трех МС, включая $\mathrm{Fe}_{40} \mathrm{Ni}_{40} \mathrm{P}_{14} \mathrm{~B}_{6}$. Однако, как отмечалось выше, недавний анализ кристаллизации это-

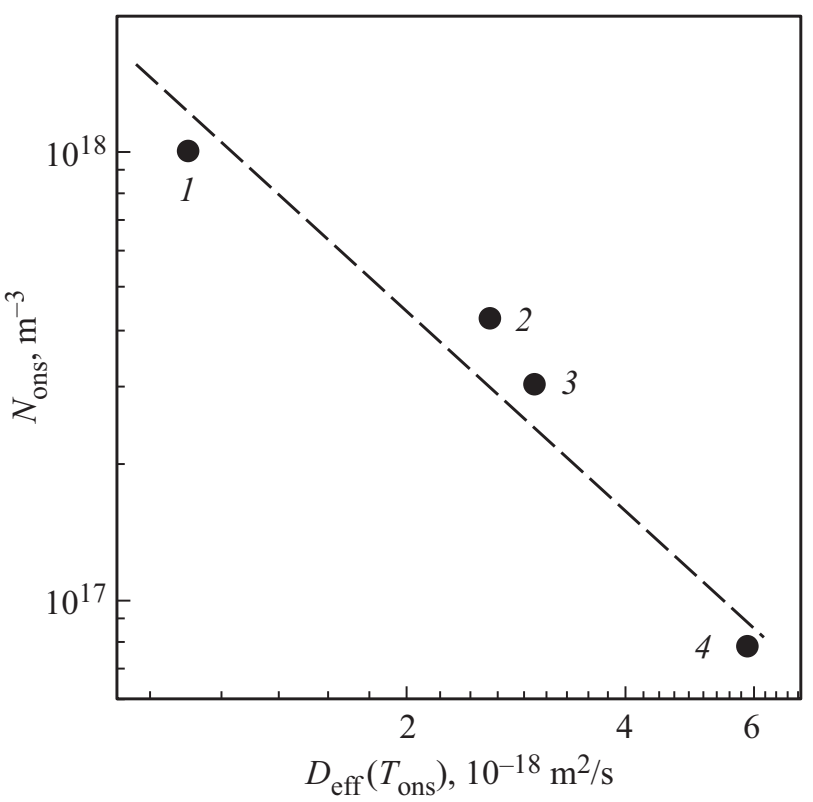

Рис. 4. Связь между пороговыми коэффициентами диффузии и объемной плотностью кристаллитов при Tons в металлических стеклах, кристаллизующихся по эвтектическому механизму. Штриховая линия - линейная аппроксимация данных, числа на графике соответствуют номерам позиций сплавов в таблице. 
го стекла [13] позволил уточнить параметры уравнения $D_{\text {eff }}(T)$, поэтому величины $D_{\text {eff }}\left(T_{\text {ons }}\right)$ и $N_{\text {ons }}$ для этого стекла были пересчитаны. Результаты расчетов, суммированные в таблице и на рис. 4 , подтверждают наличие корреляции между величинами $D_{\text {eff }}\left(T_{\text {ons }}\right)$ и $N_{\text {ons }}$ для $\mathrm{MC}$, кристаллизующихся по эвтектическому механизму. Характер этой зависимости аналогичен зависимости $N_{\text {ons }}$ от $D_{\text {eff }}\left(T_{\text {ons }}\right)$ для МC, кристаллизующихся по первичному механизму, построенной для более широкого интервала $D_{\text {eff }}$ (ons) от $1.7 \cdot 10^{-20}$ до $2.9 \cdot 10^{-18} \mathrm{~m}^{2} / \mathrm{s}[1]$. Ввиду того, что скорости диффузионно-контролируемого роста ниже, чем эвтектического, значения $N_{\text {ons }}$ первично кристаллизующихся $\mathrm{MC}$ в указанном диапазоне $D_{\text {eff }}\left(T_{\text {ons }}\right)$ выше и лежат в диапазоне $3.8 \cdot 10^{20}-1.3 \cdot 10^{24} \mathrm{~m}^{3}$. Верхняя граница диапазона $N_{\text {ons }}$ соответствует случаю нанокристаллизации - формированию наномасштабных структур, состоящих из нанокристаллов базового элемента с размерами от 8 до $30 \mathrm{~nm}$, диспергированных в остаточной аморфной матрице $[1,8]$.

Проведенный в [1] анализ для первично кристаллизующихся МС показал, что более низкие значения $D_{\text {eff }}\left(T_{\text {ons }}\right)$ свидетельствуют о снижении влияния зарождения кристаллов на термическую устойчивость аморфного состояния. Что касается эвтектически кристаллизующихся сплавов, то, несмотря на ограниченное количество экспериментальных данных (рис. 4, таблица), они согласуются с приведенной выше тенденцией. В частности, объемная плотность кристаллов в полностью закристаллизованном $\mathrm{MC} \mathrm{Fe}_{40} \mathrm{Ni}_{40} \mathrm{P}_{14} \mathrm{~B}_{6}$ при скоростях нагрева 0.02 и $0.7 \mathrm{~K} / \mathrm{s}$ составляет $3.8 \cdot 10^{18}$ и $2 \cdot 10^{18} \mathrm{~m}^{-3}$ соответственно [13], что практически на порядок превышает значение $N_{\text {ons }}$. Значительное увеличение объемной плотности кристаллитов в процессе кристаллизации свидетельствует о важной роли процесса зарождения, что согласуется с механизмом превращения, установленным для $\mathrm{Fe}_{40} \mathrm{Ni}_{40} \mathrm{P}_{14} \mathrm{~B}_{6}[1,12,21]$. Напротив, значение $N_{\text {ons }}$ в стекле $\mathrm{Fe}_{80} \mathrm{~B}_{20}$ с наиболее низким пороговым значением $D_{\text {eff }}$ практически совпадает с плотностью кристаллов в закристаллизованных образцах $\left(1.9 \cdot 10^{18} \mathrm{~m}^{3}\right)$ [10], что характерно для установленного в этом стекле механизма кристаллизации путем роста закалочных центров $[10,21]$. Безусловно, для формулирования более определенных выводов о связи величин $D_{\text {eff }}\left(T_{\text {ons }}\right)$ и $N_{\text {ons }}$ с факторами, определяющими термическую устойчивость МС, которые кристаллизуются по эвтектическому механизму, необходимы дальнейшие исследования на более широком круге сплавов, в особенности стекол, склонных к нанокристаллизации типа $\mathrm{Fe}_{33} \mathrm{Zr}_{67}$ [22].

В приведенных в таблице параметрах уравнений, описывающих температурные зависимости эффективных коэффициентов диффузии, обращает на себя внимание широкий (почти 15 порядков) диапазон эмпирически найденных значений предэкспоненциального множителя. Следует отметить, что эта особенность характерна и для аррениусовских зависимостей экспериментально измеренных коэффициентов гетеродиффузии в аморфных сплавах, значения $D_{0}$ в которых лежат в диапазоне $10^{-15}-10^{15} \mathrm{~m}^{2} / \mathrm{s}$ [23]. Природа столь широкого спектра

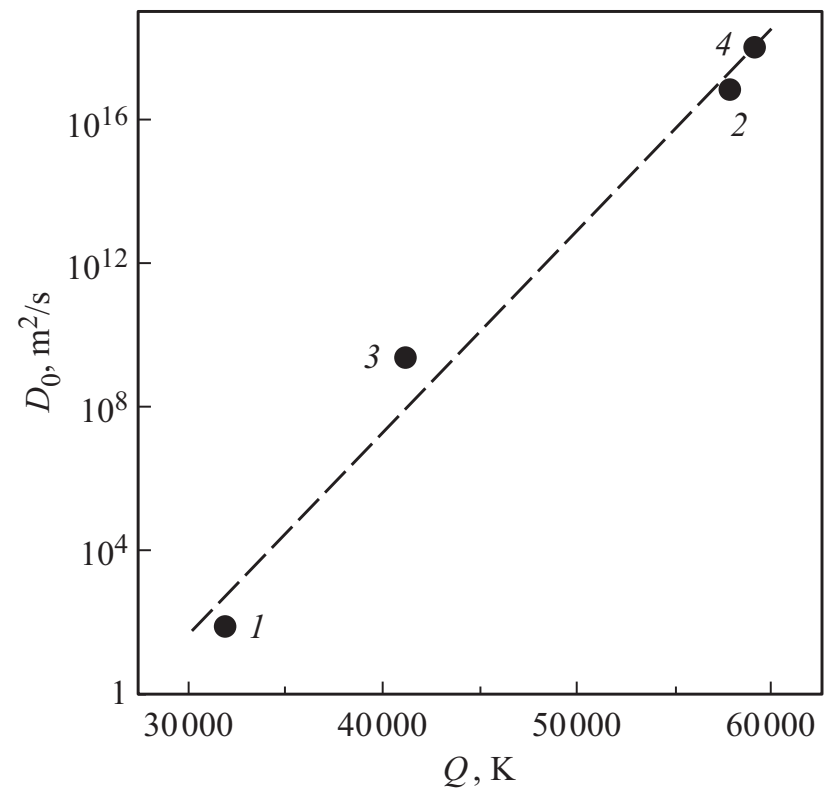

Рис. 5. Корреляция между энергиями активации и предэкспоненциальными множителями эффективных коэффициентов диффузии, контролирующей рост эвтектических колоний в металлических стеклах на основе Fе. Штриховая линия - линейная аппроксимация методом наименьших квадратов. Цифры на графике соответствуют номерам сплавов в таблице.

значений $D_{0}$ для $\mathrm{MC}$ до настоящего времени однозначно не установлена, а предположительно обусловлена особенностями коллективного характера миграции атомов в неупорядоченных структурах [23]. Одной из особенностей параметров уравнений, описывающих температурные зависимости коэффициентов диффузии в кристаллических и аморфных сплавах, является корреляция между значениями $D_{0}$ и $Q$, которая выражается соотношением $D_{0}=A \exp (Q / B)$, где $A$ и $B-$ константы. Обобщенный анализ [23] показал, что значения констант $A$ и $B$ уравнений, описывающих диффузию во всех кристаллических сплавах, составляют $2 \cdot 10^{-7} \mathrm{~m}^{2} / \mathrm{s}$ и $4527 \mathrm{~K}$ соответственно, а во всех $\mathrm{MC}-4.8 \cdot 10^{-19} \mathrm{~m}^{2} / \mathrm{s}$ и $647 \mathrm{~K}$, а различия констант обусловлены различиями механизмов диффузии в стеклах и кристаллах. Физическая природа этой корреляции, которая называется компенсационным законом, применительно к процессу диффузии заключается в том [24], что возрастание высоты энергетического барьера $(Q)$, компенсируется увеличением возможных путей для преодоления, т. е. ростом энтропийного вклада, входящего в предэкспоненциальный множитель $D_{0}$.

Несмотря на ограниченное число установленных до настоящего времени температурных зависимостей $D_{\text {eff }}(T)$, их анализ, проведенный в работе [25], показал, что значения $D_{0}$ и $Q$ также могут быть аппроксимированы приведенным выше соотношением. Однако, в отличие от результатов, приведенных в обзоре [23], в работе [25] было установлено, что параметры $A$ и $B$ уравнений $D_{\text {eff }}(T)$, характеризующих как гетеродиффузию, так и первичную кристаллизацию металлических стекол на 
основе $\mathrm{Al}$ и $\mathrm{Fe}$ различны. Более того, значения этих параметров оказались различными для эффективных коэффициентов диффузии, контролирующих первичную и эвтектическую кристаллизацию стекол на основе железа. По этой причине представлялось интересным сравнить соотношение полученных в работе параметров $D_{0}$ и $Q$ для металлического стекла $\mathrm{Fe}_{48} \mathrm{Co}_{32} \mathrm{P}_{14} \mathrm{~B}_{6}$ с аналогичными данными других стекол, приведенных в таблице. Анализ показал (рис. 5), что значения параметров $D_{0}$ и $Q$ уравнений $D_{\text {eff }}(T)$, характеризующих эвтектическую кристаллизацию стекол на основе железа, удовлетворительно аппроксимируются зависимостью $\ln D_{0}=(-34.92 \pm 6.1)+\left(1.29 \cdot 10^{-3} \pm 1.3 \cdot 10^{-4}\right) Q$. Параметры этой линейной зависимости, показанной на рис. $5,\left(A=6.83 \cdot 10^{-16} \mathrm{~m}^{2} / \mathrm{s}\right.$ и $\left.B=775 \mathrm{~K}\right)$ практически совпадают с постоянными, найденными в [25] для эвтектической кристаллизации, и гораздо ближе к параметрам, описывающим гетеродиффузию в металлических стеклах, чем в кристаллических сплавах. Поскольку основным отличием диффузионных механизмов в аморфных и кристаллических материалах является степень кооперативности диффузионного акта [23], полученный результат дает основание предположить, что переход атомов через границу раздела носит групповой, а не индивидуальный характер. Отметим, что это предположение согласуется с результатами теоретического анализа линейного роста кристаллов [3] и свидетельствует о необходимости учета этого обстоятельства в теоретических моделях процессов зарождения и роста кристаллов.

Создание адекватных теоретических подходов представляется важной проблемой, поскольку значения большинства термодинамических и кинетических параметров, контролирующих скорости зарождения и роста кристаллов в МС, определяются путем сопоставления экспериментальных данных с величинами, рассчитанными в рамках конкретных моделей. Достаточно большой разброс параметров, характеризующих эффективную диффузию, контролирующую процесс кристаллизации МС [1], обусловлен не только низкой точностью экспериментальных оценок, но и различиями моделей, в рамках которых определялись значения $D_{\text {eff. }}$ Тем не менее, как абсолютные значения $D_{\text {eff }}$, так и рассмотренные в работе корреляции открывают новые возможности для анализа процесса кристаллизации МС, установления факторов, определяющих термическую устойчивость, и, в конечном итоге, для создания научных подходов к разработке новых материалов с высоким уровнем физических свойств.

\section{4. Выводы}

Экспериментальные исследования процесса изотермического роста эвтектических колоний в $\mathrm{MC}$ $\mathrm{Fe}_{48} \mathrm{Co}_{32} \mathrm{P}_{14} \mathrm{~B}_{6}$ и анализ полученных данных позволяет сделать следующие основные выводы.

1. Установлено, что при повышении температуры отжига от 679 до $717 \mathrm{~K}$ скорость роста эвтектических колоний, оцененная по их максимальному размеру, возрастает приблизительно на два порядка величины от $3.9 \cdot 10^{-11}$ до $3.6 \cdot 10^{-9} \mathrm{~m} / \mathrm{s}$.

2. Показано, что эффективные коэффициенты диффузии на межфазной границе, контролирующие линейный рост в исследованном диапазоне температур возрастают от $2.04 \cdot 10^{-20}$ до $2.02 \cdot 10^{-18} \mathrm{~m}^{2} / \mathrm{s}$ и хорошо аппроксимируются уравнением типа Аррениуса $\ln D_{\text {eff }}=\exp (41.46 \pm 4)-(58984 \pm 2836) / T$.

3. Анализ начальных стадий кристаллизации МC $\mathrm{Fe}_{48} \mathrm{Co}_{32} \mathrm{P}_{14} \mathrm{~B}_{6}$ в условиях нагрева со скоростью $0.17 \mathrm{~K} / \mathrm{s}$ показал, что значение эффективного коэффициента при температуре начала кристаллизации $(727 \mathrm{~K})$ составляет $5.9 \cdot 10^{18} \mathrm{~m}^{2} / \mathrm{s}$, а экспериментально определенная доля закристаллизованного объема 0.055 образована кристаллитами, средний размер которых составляет $1.1 \mu \mathrm{m}$, а объемная плотность $-7.9 \cdot 10^{16} \mathrm{~m}^{-3}$.

4. Установлено, что параметры $\left(D_{0}\right.$ и $\left.Q\right)$ аррениусовских зависимостей $D_{\text {eff }}(T)$ для группы MC на основе $\mathrm{Fe}$, кристаллизующихся по эвтектическому механизму, включая исследуемый сплав, хорошо аппроксимируются зависимостью $\ln D_{0}=(-34.92 \pm 6.1)$ $+\left(1.29 \cdot 10^{-3} \pm 1.3 \cdot 10^{-4}\right) Q$ (компенсационный закон), параметры которой свидетельствуют о коллективном характере перехода атомов через границу раздела.

5. Сопоставление экстраполяций зависимостей $D_{\text {eff }}(T)$ в области температур кристаллизации МС $\mathrm{Fe}_{48} \mathrm{Co}_{32} \mathrm{P}_{14} \mathrm{~B}_{6}$ и $\mathrm{Fe}_{40} \mathrm{Ni}_{40} \mathrm{P}_{14} \mathrm{~B}_{6}$ показывает, что величина $D_{\text {eff }} \mathrm{FeCo}$ стекла примерно на 2-3 порядка ниже, чем стекла на железоникелевой основе, что является основной причиной различий термической устойчивости аморфной структуры в этих стеклах.

\section{Конфликт интересов}

Авторы заявляют, что у них нет конфликта интересов.

\section{Список литературы}

[1] В.И. Ткач, Е.А. Свиридова, С.В. Васильев, О.В. Коваленко. ФМM 118, 806 (2017).

[2] J.W. Cahn, W.B. Hillig, G.W. Sears. Acta Met. 12, 1421 (1964).

[3] L.V. Mikheev, A.A. Chernov. J. Cryst. Growth 112, 591 (1991).

[4] W.-L. Chan, R.S. Averback, Y. Ashkenazy. Phys. Rev. B 82, 020201 (2010).

[5] U. Köster, U. Herold, H.-G. Hillerbrand, J. Denis. J. Mater. Sci. 15, 2125 (1980).

[6] H.B. Aaron, D. Fainstein, G.R. Kotler. J. Appl. Phys. 41, 4404 (1970).

[7] D.G. Morris. Scr. Metallurg. 16, 585 (1982).

[8] Г.Е. Абросимова, А.С. Аронин, О.И. Баркалов, М.М. Дементьева. ФТТ 55, 1665 (2013).

[9] Дж. Кристиан. Теория превращений в металлах и сплавах. Мир, М. (1978). 806 с.

[10] A.L. Greer. Acta Metallurg. 30, 171 (1982).

[11] P.M. Anderson, A.E. Lord jr. J. Non-Cryst. Solids 37, 219 (1980). 
[12] V.V. Popov, V.I. Tkatch, S.G. Rassolov, A.S. Aronin. J. NonCryst Solids 356, 1344 (2010).

[13] S.V. Vasiliev, O.V. Kovalenko, K.A. Svyrydova, A.I. Limanovskii, V.I. Tkatch. J. Mater. Sci. 54, 5788 (2019).

[14] V.I. Tkatch, S.G. Rassolov, V.V. Popov, V.Yu. Kameneva, O.A. Petrenko. Mater. Lett. 58, 2988 (2004).

[15] S.A. Kostyrya, B. Idzikowski, V.I. Tkatch, V.V. Popov, S.G. Rassolov. Phys. Status Solidi B 243, 339 (2006).

[16] В.И. Парфений, Е.А. Свиридова, С.В. Васильев, В.И. Ткач. Тр. Кольского НЦ РАН. Химия и материаловедение 10, 270 (2019).

[17] C.V. Thompson, F. Spaepen. Acta Metallurg. 22, 1855 (1979).

[18] K.F. Kelton. In: Solid State Phys. Advances in Research and Application / Eds H. Ehrenreich, D.Turnbull. Acad. Press, N. Y. (1991). P. 75.

[19] H.E. Kissinger. J. Res. Nature Bur. Stand. 57, 217 (1956).

[20] C.V. Thompson, A.L. Greer, F. Spaepen. Acta Metallurg. 31, 1883 (1983).

[21] V.I. Tkatch, A.I. Limanovskii, V.Yu. Kameneva. J. Mater. Sci. 32, 5669 (1997).

[22] N.X. Sun, K. Zhang, X.H. Zhang, X.D. Liu, K. Lu. Nanostruct. Mater. 7, 637 (1996).

[23] F. Faupel, W. Frank, M.-P. Macht, H. Mehrer, V. Naundorf, K. Ratzke, H.R. Shrober, S.K. Sharma, H. Teichler. Rev. Mod. Phys. 75, 237 (2003).

[24] Г.Б. Федоров. В сб.: Подвижность атомов в кристаллической решетке / Под ред. В.Н. Свечникова. АН УССР, Киев (1965). C. 40.

[25] A. Yelon, B. Movaghar, H.M. Branz. Phys. Rev. B 46, 12244 (1992).

[26] O.V. Kovalenko, S.V. Vasiliev, V.I. Tkatch. J. Non-Cryst. Solids 518, 36 (2019)

Редактор К.В. Емиев 